\title{
Effect of synthetic hormones on reproduction in Mastomys natalensis
}

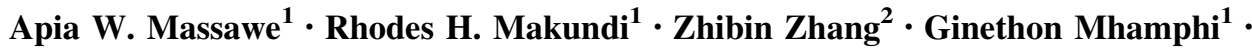 \\ Ming Liu ${ }^{3}$ Hong-Jun $\mathrm{Li}^{2} \cdot$ Steven R. Belmain ${ }^{4}$
}

Received: 12 January 2017/Revised: 31 May 2017/Accepted: 3 June 2017/Published online: 14 August 2017

(C) The Author(s) 2017. This article is an open access publication

\begin{abstract}
Rodent pest management traditionally relies on some form of lethal control. Developing effective fertility control for pest rodent species could be a major breakthrough particularly in the context of managing rodent population outbreaks. This laboratory-based study is the first to report on the effects of using fertility compounds on an outbreaking rodent pest species found throughout sub-Saharan Africa. Mastomys natalensis were fed bait containing the synthetic steroid hormones quinestrol and levonorgestrel, both singly and in combination, at three concentrations (10,50,100 ppm) for 7 days. Consumption of the bait and animal body mass was mostly the same between treatments when analysed by sex, day and treatment. However, a repeated measures ANOVA indicated that quinestrol and quinestrol + levonorgestrel treatments reduced consumption by up to $45 \%$, particularly at the higher concentrations of 50 and $100 \mathrm{ppm}$. Although there was no clear concentration effect on animal body mass, quinestrol and quinestrol + levonorgestrel lowered body
\end{abstract}

Communicated by J. Jacob.

Steven R. Belmain

s.r.belmain@gre.ac.uk

1 Pest Management Centre, Africa Centre of Excellence for Innovative Rodent Pest Management and Biosensor Technology Development, Sokoine University of Agriculture, Morogoro, Tanzania

2 State Key Laboratory of Pest Management of Insects and Rodents, Institute of Zoology, Chinese Academy of Sciences, Beijing 100101, China

3 State Key Laboratory of Reproductive and Stem Cell Biology, Institute of Zoology, Chinese Academy of Sciences, Beijing 100101, China

4 Natural Resources Institute, University of Greenwich, Chatham Maritime, Kent ME4 4TB, UK mass by up to $20 \%$ compared to the untreated and levonorgestrel treatments. Quinestrol and quinestrol + levonorgestrel reduced the weight of male rat testes, epididymis and seminal vesicles by $60-80 \%$, and sperm concentration and motility were reduced by more than $95 \%$. No weight changes were observed to uterine and ovarian tissue; however, high uterine oedema was observed among all female rats consuming treated bait at 8 and 40 days from trial start. Trials with mate pairing showed there were significant differences in the pregnancy rate with all treatments when compared to the untreated control group of rodents.

Keywords Contraceptive bait - Fertility control . Levonorgestrel · Multimammate rat · Quinestrol

\section{Key message}

- Contraceptive hormones quinestrol and levonorgestrel were able to limit the reproductive capacity of the most common rodent pest species found across sub-Saharan Africa, the multimammate rat Mastomys natalensis.

- Consumption rates of bait treated with the hormones were similar between males and females and to that observed in the untreated control, with no significant effects on body mass.

- Pregnancy rates were much lower among paired animals where one or both sexes consumed contraceptive hormones in comparison with untreated pairs.

\section{Introduction}

Rodent population outbreaks are well known to occur in many parts of the world where such outbreaks are caused by a variety of abiotic, biotic and anthropogenic factors 
(Singleton et al. 2010). The multimammate rats, genus Mastomys, are indigenous to sub-Saharan Africa where population outbreaks have been documented since the early nineteenth century (Fiedler 1988; Leirs 1995). Rodent population outbreaks resulting in high agricultural losses in eastern and southern Africa are largely attributed to Mastomys natalensis (Leirs et al. 1994; Mwanjabe et al. 2002; Makundi and Massawe 2011). M. natalensis are considered a threat to many cereal crops (Mulungu et al. 2011) with economic losses especially high among small holder farmers (Stenseth et al. 2003; Makundi et al. 2005; Makundi and Massawe 2011). M. natalensis are also reservoirs of several zoonotic diseases of public health concern (Meerburg et al. 2009; Katakweba et al. 2012; Bordes et al. 2015; Morand et al. 2015). There are several abiotic and biotic explanatory factors associated with outbreaks of M. natalensis in Africa (Leirs 1992; Massawe et al. 2011). Outbreaks of M. natalensis seem to originate locally (Leirs et al. 1994) and are associated with rainfall patterns (Leirs et al. 1997). When environmental factors (e.g. prolonged rainfall, early onset rain, increased vegetation cover) are favourable, rapid reproduction occurs, enabling $M$. natalensis to build up in numbers within a relatively short time period (Leirs 1995).

Rodent management strategies in agro-ecological systems vary according to which rodent pest species are present, crop type, method of cropping, and the availability, affordability and cost-benefit of rodent management methods (Singleton and Petch 1994). Rodent control methods largely rely on the use of rodenticides (Buckle and Smith 2015). Both acute and chronic rodenticides have been used extensively during rodent outbreaks (Brown et al. 1997, 2002; Ngowo et al. 2005). Through increased rates of recruitment, rodent populations can rapidly recover after efforts to reduce populations through poisoning, trapping, hunting and other mortality-focussed population management strategies (Singleton et al. 2007). As described by Stenseth et al. (2001), the use of mortality control results in increased reproductive compensation and survival within short-lived, fast breeding animals such as rodents; thus populations rapidly recover. In contrast to mortality-based control options, the use of fertility control has been argued to be more ecologically sound (Stenseth et al. 2001) whereby infertile animals can remain in the population, therefore sustaining density dependant feedback to recruitment and survival (Zhang 2000). However, some compensation at the population level can still occur through higher survival of juveniles (Jacob et al. 2004; Williams et al. 2007). Managing populations through limiting fertility has also been argued to be more humane (Barlow 2000), and more safe and cost effective (Hone 1992) than the use of mortality control.

Fertility limiting compounds can cause permanent or temporary sterility in either sex, reduce the number of offspring or impair the fertility of offspring produced (Humphrys and Lapidge 2008) through a reduction in either fertility or fecundity (Bomford 1990). Several antifertility compounds have been used in controlling reproduction in various animal species, through contraception or sterilization (Kirkpatrick and Turner 1985; Tuyttens and Macdonald 1998; Fagerstone 2002; Jacob et al. 2008; Massei and Cowan 2014). The most common approach to wildlife contraception has been through the use of steroid hormones, particularly natural and synthetic oestrogens, progestins and androgens (Massei and Cowan 2014). Other chemical types such as alpha-chlorohydrin (sterilant) and bromocriptine (enzyme inhibitor of prolactin) have also been used for wildlife management (Sridhara and Dubey 2006). Synthetic progestins such as norgestomet, melengestrol acetate, megestrol acetate and levonorgestrel have been widely used in zoo animals, livestock and wildlife (Nave et al. 2002). In general, practical use of fertility control often faces problems with poor palatability and repeated baiting of contraceptive compounds.

Zhang (2015) demonstrated that quinestrol and levonorgestrel delivered at low dosage $(10 \mathrm{ppm})$ could deliver long-term anti-fertility effects in several wild rodent species. Several studies have indicated that a single baiting of quinestrol and/or levonorgestrel at a dosage of 10-50 $\mu \mathrm{g} /$ $\mathrm{ml}(0.001-0.005 \%)$ delivered at the start of the rodent breeding season can successfully limit breeding for 1-2 years (Zhang 2015). Levonorgestrel is a progesterone analogue used as an emergency contraceptive to prevent pregnancy by preventing or interrupting ovulation and egg implantation in humans and other animals (GemzellDanielsson and Marions 2004; Novikova et al. 2007). It affects the cervical mucus or the ability of the sperm to bind to the egg (Asa and Porton 2005). It is still unclear whether levonorgestrel has effects on fertilization or implantation (Novikova et al. 2007). Quinestrol is a synthetic oestrogen homologue employed in many long-term oral contraceptives for human use (Zhao et al. 2007). Little is known regarding the effect of quinestrol on male fertility (Massei and Cowan 2014). Trials with quinestrol and levonorgestrel have shown some population limiting effects with rodent species such as Brandt's voles ( $\mathrm{La}$ siopodomys brandtii), Mongolian gerbils (Meriones unguiculatus) and plateau pikas (Ochotona curzoniae) (Zhao et al. 2007; Wang et al. 2011; Liu et al. 2012, 2016; Lv and Shi 2012; Qu et al. 2015). Anti-fertility effects of these compounds appear to impair reproductive performance of rodents by reducing the size and function of male reproductive organs, interfering with spermatogenesis, decreasing sperm concentration and motility and reducing female pregnancy rates and litter size through inducing uterine oedema (Wang et al. 2011; Lv and Shi 2012; Fu et al. 2013). Both compounds decompose quickly under 
field conditions, with half-lives of 5-16 days in soil and less than 3 days in water (Tang et al. 2012a, b; Zhang et al. 2014). Non-target effects on birds appear to be minimal (Qu et al. 2015).

The current study evaluates the potential effects of levonorgestrel and quinestrol on bait consumption and reproductive performance of Mastomys natalensis to determine whether the compounds are sufficiently palatable and have negative effects on reproductive potential of this rodent species. Our laboratory-based study focused on four aspects: (1) bait palatability; (2) effects on body and reproductive organ weight; (3) physiological changes in reproductive organs and cells; and (4) effects on birth rate and litter size.

\section{Materials and methods}

\section{Experimental animals}

The study was conducted in laboratories of the Pest Management Centre, Sokoine University of Agriculture, Morogoro, Tanzania (6 $\left.50^{\prime} 42.66^{\prime \prime} \mathrm{S}, 37^{\circ} 39^{\prime} 29.14^{\prime \prime} \mathrm{E}\right)$. Wild live captured adult Mastomys natalensis were used in the laboratory experiments. Animals were captured using Sherman LFA aluminium traps (H.B. Sherman Traps, Tallahassee, Florida) baited with peanut butter mixed with coarse maize flour in fallow agricultural fields owned by Sokoine University of Agriculture, which granted permission for the trials to take place. A total of 316 animals (158 males and 158 females) were captured between 25 September and 27 October 2015 and caged separately for at least two weeks to acclimatize to laboratory conditions (12:12 h light/dark, $24-30{ }^{\circ} \mathrm{C}, \quad 40-60 \% \mathrm{rh}$ [relative humidity]) before the baiting experiments were conducted. Captured animals were weighed and sexed, and animals of approximately the same weight $(37 \pm 5 \mathrm{~g})$ were used for all trials, ensuring equal mean weights between sexes. All animals were fed ad libitum on standard pellet bait (see below) and water with wood shavings for nesting material. Female animals with a closed vagina at the time of capture were used for all trials.

\section{Bait preparation}

Ten kilograms of maize flour was combined with $250 \mathrm{~g}$ of fish meal to give a $2.5 \% \mathrm{w} / \mathrm{w}$ fish meal in maize flour admix. The maize/fish meal flour was mixed with 201 of boiling water and cooked for $15 \mathrm{~min}$ while being continuously stirred to form a stiff paste; the paste was left to cool to room temperature. The base rodent bait is then made by thoroughly mixing two-thirds roughly crushed maize $(6.66 \mathrm{~kg})$ and one-third of the maize/fish meal flour
$(3.33 \mathrm{~kg})$. The bait is then passed through a mechanical pelletiser (NMG-744, Nikai Mfg., United Arab Emirates), to provide $10 \mathrm{~kg}$ of standard rodent bait.

In order to make contraceptive baits, powdered quinestrol and levonorgestrel (Beijing Zizhutiangong Science and Technology Ltd, China) were weighed in 0.1, 0.5 and $1 \mathrm{~g}$ quantities to prepare rodent bait at concentrations of 10,50 and $100 \mathrm{ppm}$, respectively, in $10 \mathrm{~kg}$ of standard rodent bait. For the quinestrol + levonorgestrel combination, the compounds were mixed equally at a ratio of $1: 1$. Each quantity of contraceptive was dissolved in $100 \mathrm{ml}$ of ethanol at $60-70{ }^{\circ} \mathrm{C}$. The ethanol-contraceptive solution was then mixed with a sugar solution made from $200 \mathrm{~g}$ sucrose in $1000 \mathrm{ml}$ water. This sugar-contraceptive solution was thoroughly mixed with the crushed maize just before adding the maize flour and fish meal paste. The plain bait was similarly prepared without contraceptives. All pelletized baits were dried in the shade at ambient temperature and stored in dark dry conditions until required.

\section{Bait acceptance and weight loss}

Bait acceptance was evaluated using 50 males and 50 females adult $M$. natalensis. Each animal was kept in a separate animal cage and provided with $10 \mathrm{~g}$ of plain bait (control) or bait containing different concentrations of contraceptives $(10,50$ and $100 \mathrm{ppm}$ for each treatment of quinestrol, (QE), levonorgestrel (LNG) or quinestrol + levonorgestrel (QE + LNG), with five animals of each sex per treatment. Each animal was provided with fresh bait (10 g) every day for seven consecutive days. Water was supplied ad libitum for each animal. The body weight of each animal was recorded before feeding with the contraceptive or plain bait and thereafter daily for seven days. The amount of bait consumed daily was determined by subtracting the amount remaining from the original weight (10 g) of bait provided. All baits were weighed $24 \mathrm{~h}$ after removal from the cage to allow them to dry in case of urine contamination affecting bait weight. The percentage consumption of bait by weight was calculated.

\section{Reproductive physiology}

A total of 47 males and 49 females were used for histological observations of the reproductive organs using animals fed on contraceptive bait for seven days. Animals were anaesthetized using diethyl ether and killed by cervical dislocation on day eight. On dissection, female and male reproductive organs were observed in situ to note any abnormalities, e.g. uterine oedema. The uterus, ovaries, testes, epididymis and seminal vesicles were removed and 
weighed. The epididymis of each male animal was dissected in a glass Petri dish containing $1 \mathrm{ml}$ of $0.85 \%$ normal saline. A drop of the suspension was examined under magnification for sperm motility observations using an ordinary light microscope at magnification $20 \times$. Another drop of the suspension was used to prepare a smear for sperm morphology analysis according to WHO standard protocols (WHO 2010). Smears were air dried and fixed with a solution of diethyl ether and ethanol (50:50) for $30 \mathrm{~min}$. The smears were stained using $10 \%$ Giemsa for 30 min, washed with running tap water, dried and examined under oil immersion at magnification $100 \times$ to assess the sperm morphology. Two hundred sperm were observed per slide, and the number of sperm with abnormalities was recorded and expressed as percentage abnormal. In order to carry out sperm counts, the remaining epididymis samples were placed in glass test tubes and kept at $4{ }^{\circ} \mathrm{C}$ for $2 \mathrm{~h}$ in order to release the sperm. The samples were then diluted 1:10 by adding $9 \mathrm{ml}$ of distilled water and placed in a modified Fuchs Rosenthal (B.S.748) chamber, following the WHO standard protocol to count sperm (WHO 2010).

\section{Effects on birth rate and litter size}

A total of 216 animals (108 male and 108 female adult $M$. natalensis) were used to determine the potential effects of the contraceptives on pregnancy rate and litter size. Animals were provided with $10 \mathrm{~g}$ of contraceptive bait at three different concentrations (10, 50 and $100 \mathrm{ppm})$, while control animals were fed $10 \mathrm{~g}$ of plain bait. The bait was delivered for seven consecutive days, and water was provided ad libitum. Each concentration of the bait was provided to three replicates of male and female animals for each of the two fertility compounds and their combination. After the seven days of baiting, animals were paired for 10 days in four combinations. Each of the three treated females per group was paired with either a treated or untreated male, and each of the untreated females per group was paired with a treated or untreated male. Thereafter, females were retained and fed plain bait for 30 days for observation of pregnancy and litter size. The number of pups born in each litter produced by pregnant females was counted, weighed and compared with the control batch. Females that were not pregnant from all treatments including controls (untreated females paired with untreated males) were killed after 40 days from the start of the trial for uterus and ovary observation. The uteri and ovaries were dissected, weighed with all normal and abnormal features noted.

\section{Data analysis}

Statistical analyses were performed using XLSTAT version 2015.1.03.16409 (Addinsoft, Paris, France).
Comparisons of organ weights and pregnancy rates were made by an analysis of variance (ANOVA) with Duncan's Multiple Range Test to separate the means at the $95 \%$ confidence interval. Bait uptake and body mass comparisons were made with a repeated measures (using daily measurements) ANOVA (least squares) followed by Duncan's MRT.

\section{Results}

\section{Bait acceptance and weight loss}

The mean consumption of bait by rodents when individually grouped by sex, treatment and day was generally the same, with a few minor significant differences and general trends to be noted. (Table 1). Both male and female rodents fed quinestrol at $100 \mathrm{ppm}$ consumed less bait on day 1 compared to the control group; however, all other treatment groups did not significantly vary from the control on a daily basis. Using daily measures of consumption in a repeated measures analysis of variance showed that there was no significant difference in the consumption rate between females (2.88 g/day) and males $(3.03 \mathrm{~g} /$ day $) \quad(F=0.389, \quad d f=1, \quad P=0.533)$. However, consumption did vary by treatment with $\mathrm{QE}$ (2.24 g/day) and QE + LNG (2.20 g/day) showing reduced consumption compared to the control (4.0 g/day), with consumption in the LNG (3.74 g/day) treatment similar to the control $(F=21.856 d f=3$, $P<0.0001)$. Increasing concentration also had an effect on consumption whereby bait consumption with the $10 \mathrm{ppm}$ treatment $(2.95 \mathrm{~g} /$ day $)$ was not different from the untreated control (3.35 g/day) but where $50 \mathrm{ppm}$ (2.76 g/day) and $100 \mathrm{ppm}(2.75 \mathrm{~g} /$ day $)$ showed significantly less bait consumption compared to the untreated bait $(F=4.600, d f=2, P=0.010)$. Similarly on a daily basis, body mass of animals in different treatments did not significantly vary in comparison with the control group (Table 2). In a few instances, body mass dropped significantly for certain treatment groups. For example, males fed QE + LNG at $50 \mathrm{ppm}$ had a significantly lower body mass from day 3 to 7 , although no significant change was observed at the 10 and $100 \mathrm{ppm}$ rates for males fed with the QE + LNG bait. A repeated measures ANOVA indicated that the sex $(F=0.202, d f=1$, $P=0.653)$ of the animals and the concentration ( $F=0.447, d f=2, P=0.639)$ of the bait had no effect on body mass, whereas the treatment $(F=3.795, d f=3$, $P=0.010)$ showed that the $\mathrm{QE}+\mathrm{LNG}$ treatment had the lowest mean body mass $(32.43 \mathrm{~g})$, followed by QE (35.27 g), with the control (38.99 g) and LNG (38.92 g) treatments showing no difference from each other. 


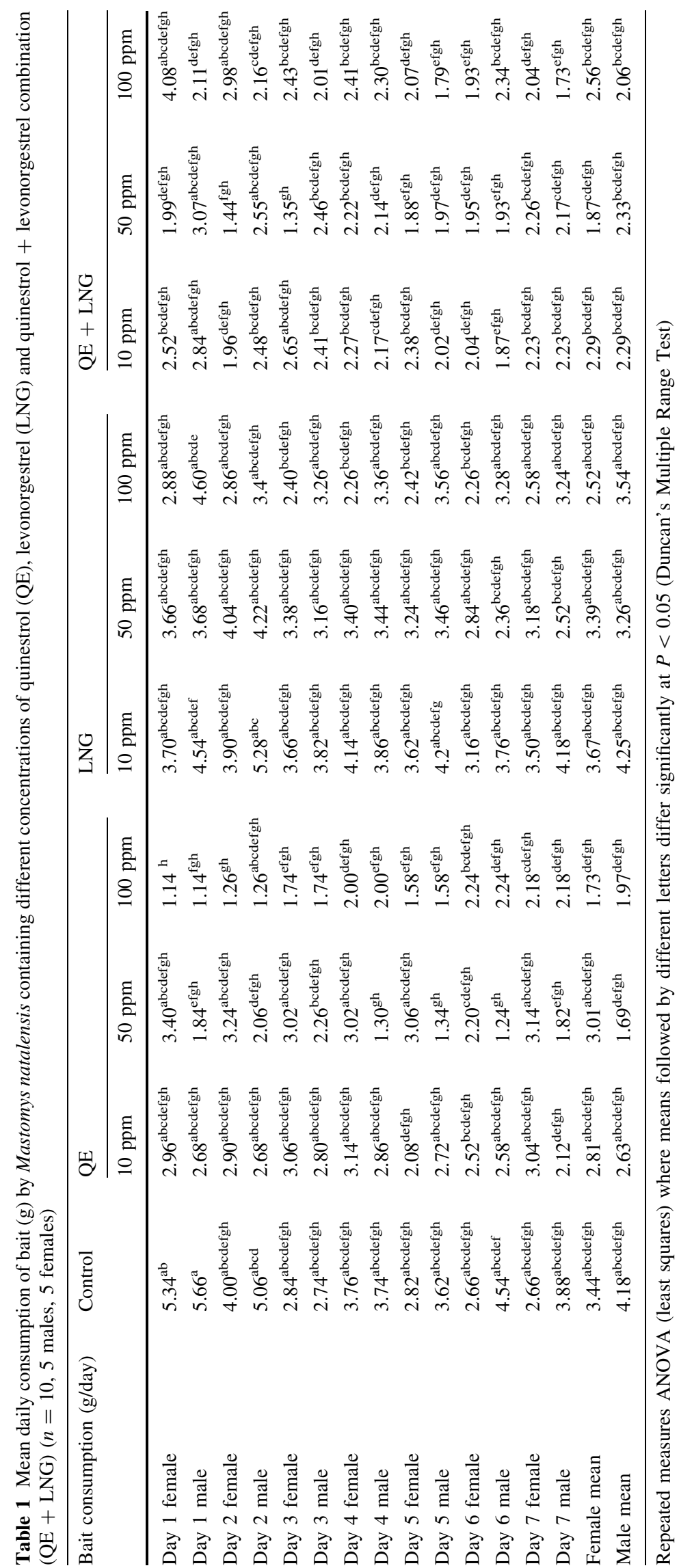




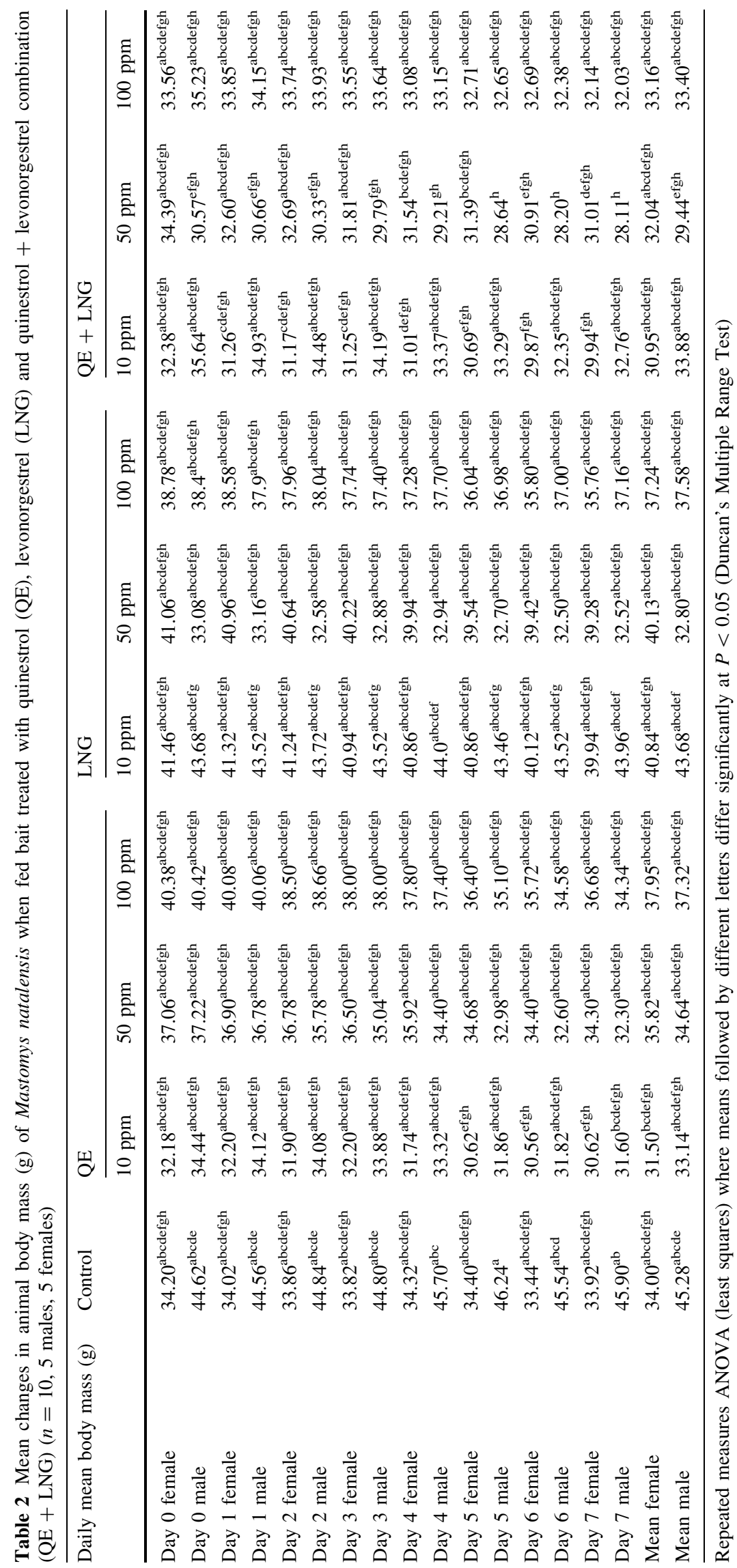




\section{Reproductive physiology}

On dissection, all males and females were considered to be sexually mature; with all males the testes were fully descended, and with females all uteri were fully vascularized. There was no difference in uterine or ovarian weights $(P>0.05)$ or structure except for a marked uterine oedema in animals feeding on bait treated with quinestrol and quinestrol + levonorgestrel at all concentrations, both for females killed at 8 and 40 days from trial start (Fig. 1). Effects of hormone treatments on testis weight, epididymis weight and sperm count were significant. On its own, levonorgestrel did not reduce the weight of the testes and epididymis but did have an effect on the seminal vesicle weight (Table 3), whereas quinestrol did not have a significant effect on the weight of the epididymis but did lower the weight of the testes and seminal vesicle. The effects of the two hormones together suggest that most of the organ weight reduction can be attributed to quinestrol. However, the QE + LNG combination does appear to have advantages in terms of reducing sperm motility below that achieved by either compound on its own (Table 3). The weight of seminal vesicles of all animals feeding on bait treated with contraceptive treated was lower $(F=11.55$, $d f=9, \quad P<0.0001)$ than that of the control group (Table 3). The lowest weight of seminal vesicles was observed in animals feeding on bait treated with QE and $\mathrm{QE}+$ LNG. Sperm concentration and motility decreased
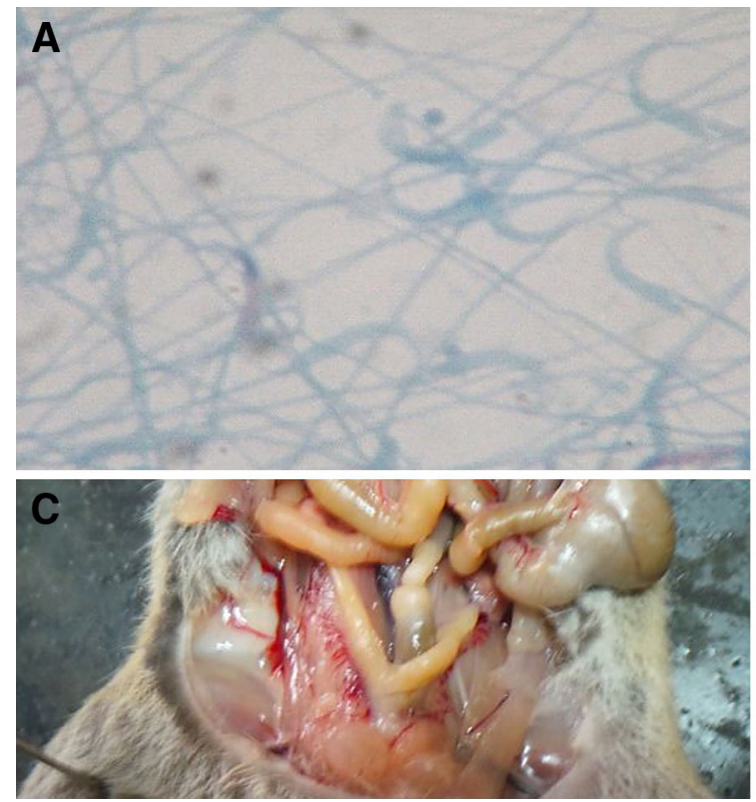

Fig. 1 Physiological effect on male and female Mastomys natalensis when feeding on bait treated with fertility compounds. a Normal sperm when males fed on untreated bait $(400 \times$ magnification $)$; b Sperm morphology in male M. natalensis when fed on quinestrol $(\mathrm{QE})$, or quinestrol + levonorgestrel combination $(\mathrm{QE}+\mathrm{LNG})$ bait significantly $\quad(F=13.18, \quad d f=9, \quad P<0.0001, \quad$ and $F=25.34, \quad d f=9, \quad P<0.0001) \quad$ in treated animals (Table 3). A significant $(F=22.10, d f=8, P<0.0001)$ increase of abnormal morphology of sperm occurred in animals feeding on bait treated with $\mathrm{QE}$ and $\mathrm{QE}+\mathrm{LNG}$ but not with LNG alone compared to the control group (Fig. 1).

\section{Effects on birth rate and litter size}

Time to delivery was similar for all treatments (23 \pm 3 days). For QE and QE + LNG treatments, pregnancy and litter size were significantly reduced $(F=20.17, \quad d f=3, \quad P<0.0001 ; \quad F=16.22, \quad d f=3$, $P<0.0001$, respectively) in animals fed on treated bait that were paired with those of either sex fed on untreated or treated bait compared to the control (untreated males with untreated females) (Table 4). There were no pregnancies in females paired with treated males or when both sexes were fed on treated bait. When both sexes were left untreated, the average litter size was four pups.

\section{Discussion}

Rodent bait containing quinestrol and/or levonorgestrel was consumed by $M$. natalensis at a lower rate when compared to untreated bait. These findings are in contrast

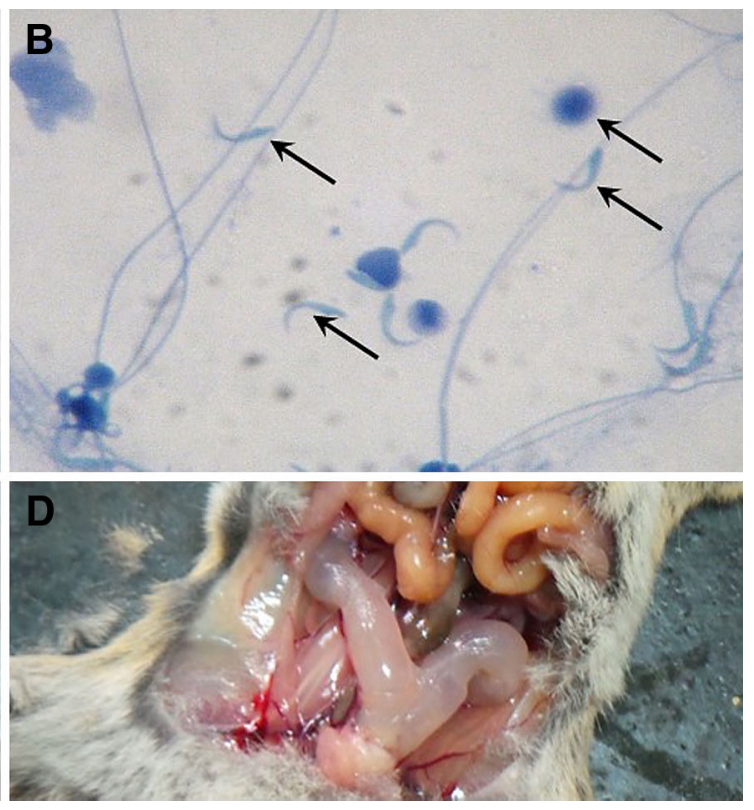

showing, at arrows, sperm with head and tail separated $(400 \times$ magnification); c Normal uterus in females fed on untreated bait. $\mathbf{d}$ Uterine oedema typically observed in female $M$. natalensis when fed on QE or $\mathrm{QE}+\mathrm{LNG}$ treated bait at 8 and 40 days from trial start 


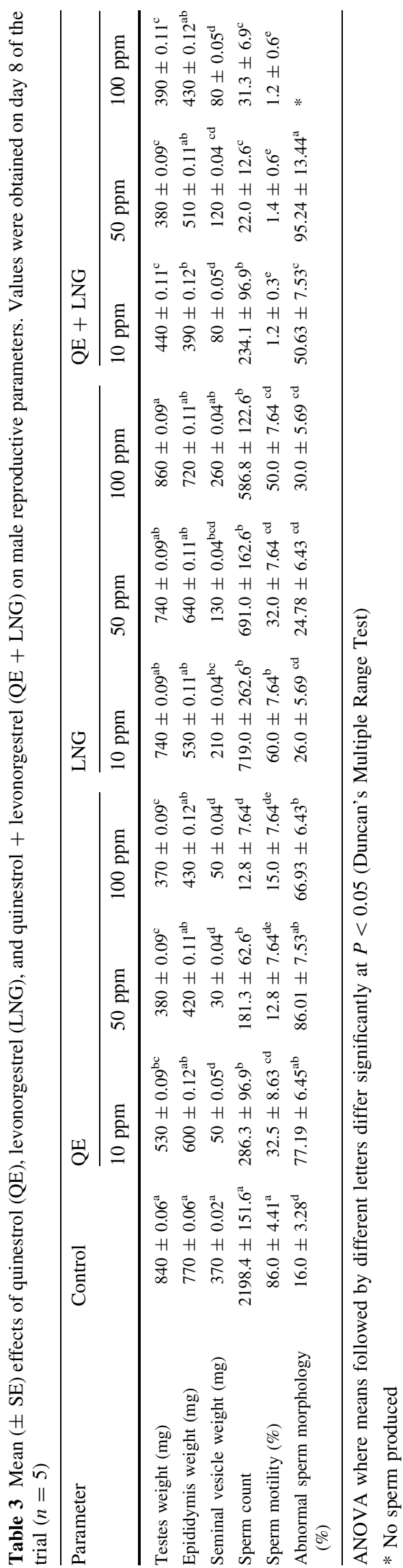

with other studies that showed no significant differences in bait consumption were associated with the concentration of fertility compounds in baits fed to other species of rodent. For example, Liu et al. (2012) showed that there were no significant differences caused by the concentration of quinestrol and levonorgestrel in bait fed to plateau pikas. Wang et al. (2011) observed no significant differences between treated and control groups on bait uptake by Brant's voles (Lasiopodomys brandtii). The loss of weight in animals feeding on bait treated with fertility compounds could be attributed to low feed intake. Similar results have been reported in Mongolian gerbils (Meriones unguiculatus) (Lv and Shi 2011, 2012) after consuming bait containing quinestrol but not after consuming levonorgestrel. However, Liu et al. (2013) reported that quinestrol had no effects on the body weight of Rattus nitidus of either sex over seven days of treatment. In the current study, animals treated with quinestrol and levonorgestrel alone experienced much lower weight loss than those treated with the quinestrol + levonorgestrel combination. Although we cannot entirely discount the possibility that observed weight loss in some treatments affected reproductive success in our trial, on dissection all male testes were fully descended and all female uteri were considered to be sexually mature by level of vascularization. As female $M$. natalensis are known to successfully reproduce at body mass levels as low as $27 \mathrm{~g}$ (Coetzee 1965), we argue that all animals were sexually mature. Furthermore, pairing studies were performed with animals fed on plain bait, which may mitigate any physiological effects of weight loss experienced during the prior 7 days feeding on treated bait. More research using feeding choice tests and histological examination of ovarian and uterine tissues could help separate nutritional effects from hormonal effects.

In the current study, quinestrol and levonorgestrel had some effects on the reproductive status of both male and female $M$. natalensis. The results demonstrated that after 7 days of bait consumption, the weight of male reproductive organs decreased, with some differences depending on treatment. On its own, levonorgestrel did not reduce the weight of the testes and epididymis but did have an effect on the seminal vesicle weight, whereas quinestrol did not have a significant effect on the weight of the epididymis but did lower the weight of the testes and seminal vesicle. The effects of the two hormones together suggest most of the organ weight reduction can be attributed to quinestrol. However, the QE + LNG combination does appear to have advantages in terms of reducing sperm motility below that achieved by either compound on its own. This demonstrates that quinestrol and levonorgestrel have anti-fertility effects on male $M$. natalensis. Among the treatments, quinestrol and quinestrol + levonorgestrel at 10, 50 and $100 \mathrm{ppm}$ were the most effective in reducing 
Table 4 Mean pregnancy and litter size per pregnancy with respect to animals fed with quinestrol $(\mathrm{QE})$, levonorgestrel (LNG) and

quinestrol + levonorgestrel $(\mathrm{QE}+\mathrm{LNG})$ treatments at three concentrations $(10,50$, $100 \mathrm{ppm}$ ) when put into mated pairs (UNFUNM-untreated female paired with untreated male, TFUNM-treated female paired with untreated male, UNFTM-untreated female paired with treated male, TFTM-treated female paired with treated male)

\begin{tabular}{|c|c|c|c|}
\hline Activity & $n$ & Mean pregnant females & Mean litter size/pregnancy \\
\hline \multicolumn{4}{|l|}{ Treatment } \\
\hline LNG & 36 & $0.36 \pm 0.07^{\mathrm{a}}$ & $2.4 \pm 0.6^{\mathrm{a}}$ \\
\hline $\mathrm{QE}+\mathrm{LNG}$ & 36 & $0.2 \pm 0.06^{\mathrm{b}}$ & $1.4 \pm 0.5^{\mathrm{b}}$ \\
\hline QE & 36 & $0.1 \pm 0.03^{\mathrm{b}}$ & $0.4 \pm 0.3^{\mathrm{c}}$ \\
\hline \multicolumn{4}{|l|}{ Concentration (ppm) } \\
\hline 100 & 36 & $0.2 \pm 0.07^{\mathrm{b}}$ & $1.9 \pm 0.6^{\mathrm{b}}$ \\
\hline 50 & 36 & $0.1 \pm 0.05^{\mathrm{b}}$ & $1.2 \pm 0.5^{\mathrm{b}}$ \\
\hline 10 & 36 & $0.2 \pm 0.06^{\mathrm{b}}$ & $1.1 \pm 0.4^{\mathrm{b}}$ \\
\hline \multicolumn{4}{|l|}{ Male-female pairs } \\
\hline UNFUNM & 27 & $0.4 \pm 0.09^{\mathrm{a}}$ & $3.7 \pm 0.8^{\mathrm{a}}$ \\
\hline TFUNM & 27 & $0.2 \pm 0.08^{\mathrm{b}}$ & $1.6 \pm 0.6^{\mathrm{b}}$ \\
\hline UNFTM & 27 & $0.0 \pm 0.03^{\mathrm{bc}}$ & $0.3 \pm 0.3^{\mathrm{bc}}$ \\
\hline TFTM & 27 & $0.0 \pm 0^{\mathrm{c}}$ & $0.0 \pm 0^{\mathrm{c}}$ \\
\hline TFTM 10 ppm & 9 & $0.0 \pm 0^{\mathrm{c}}$ & $0.0 \pm 0^{\mathrm{c}}$ \\
\hline TFUNM 10 ppm & 9 & $0.2 \pm 0.1^{\mathrm{bc}}$ & $0.9 \pm 0.6^{\mathrm{b}}$ \\
\hline UNFUNM 10 ppm & 9 & $0.4 \pm 0.2^{\mathrm{a}}$ & $3.7 \pm 1.4^{\mathrm{a}}$ \\
\hline UNFTM 10 ppm & 9 & $0.0 \pm 0^{\mathrm{c}}$ & $0.0 \pm 0^{\mathrm{c}}$ \\
\hline TFTM 50 ppm & 9 & $0.0 \pm 0^{\mathrm{c}}$ & $0.0 \pm 0^{\mathrm{c}}$ \\
\hline TFUNM 50 ppm & 9 & $0.2 \pm 0.1^{\mathrm{bc}}$ & $1.9 \pm 1.2^{\mathrm{ab}}$ \\
\hline UNFUNM 50 ppm & 9 & $0.3 \pm 0.2^{\mathrm{ab}}$ & $2.9 \pm 1.4^{\mathrm{ab}}$ \\
\hline UNFTM 50 ppm & 9 & $0.0 \pm 0^{\mathrm{c}}$ & $0.0 \pm 0^{\mathrm{c}}$ \\
\hline TFTM 100 ppm & 9 & $0.0 \pm 0^{\mathrm{c}}$ & $0.0 \pm 0^{\mathrm{c}}$ \\
\hline TFUNM 100 ppm & 9 & $0.2 \pm 0.1^{\mathrm{bc}}$ & $2.1 \pm 1.4^{\mathrm{ab}}$ \\
\hline UNFUNM 100 ppm & 9 & $0.6 \pm 0.2^{\mathrm{c}}$ & $4.6 \pm 1.5^{\mathrm{a}}$ \\
\hline UNFTM 100 ppm & 9 & $0.1 \pm 0.1^{\mathrm{b}}$ & $0.9 \pm 0.8^{\mathrm{b}}$ \\
\hline
\end{tabular}

ANOVA where means followed by different letters differ significantly at $P<0.05$ (Duncan's Multiple Range Test) spermatogenesis, which has also been observed in other rodent species (O'Donnell et al. 2001). According to $\mathrm{Li}$ et al. (2014), quinestrol reduces semen quality and this may be caused by affecting processes such as sperm maturity in the epididymis and seminal vesicle secretion rates (Gonzales 2001). Various studies (Wang et al. 2011; Liu et al. 2012, 2014; Zhang 2015) have demonstrated that the concentration of these fertility compounds have significant reproductive effects on male rodents, including the greater long-tailed hamster (Tscherskia triton), Brandt's vole (Lasiopodomys brandtii), the plateau pika (Ochotona curzoniae) and laboratory mouse (Mus musculus). Although in the current study, we did not determine the effects the fertility compounds have on subsequent offspring of animals which consumed treated bait, other studies have demonstrated that offspring of mothers treated with quinestrol were infertile whereas all male and female offspring from levonorgestrel-treated mothers were fertile (Lv et al. 2012).

Our findings show that the consumption of either quinestrol alone or quinestrol + levonorgestrel at the lowest concentration of $10 \mathrm{ppm}$ for 7 days was sufficient to induce infertility in male $M$. natalensis for at least 10 days post-treatment. Although some sperm are still produced at this treatment dose, the observed reduction in sperm number and sperm quality is accepted as male infertility (WHO 2010). This has been observed in other rodent species including the greater long-tailed hamster (Zhang et al. 2005) fed on bait containing quinestrol and levonorgestrel at 10 and $30 \mathrm{ppm}$. Zhao et al. (2007) also reported that a dosage of quinestrol of $0.35 \mathrm{mg} / \mathrm{kg}$ body weight for male voles is effective to control this species in the field. According to Lv and Shi (2011), multiple dosages of $10 \mathrm{ppm}$ of quinestrol + levonorgestrel delivered at one week intervals showed higher anti-fertility effects on female Mongolian gerbils than a single dosage treatment.

In female $M$. natalensis treated with quinestrol, uterine oedema was observed both at 8 days and 40 days, i.e. up to 33 days post-treatment. This has been attributed to abnormal amounts of oestrogen and progesterone which has been observed to lead to structural changes of the uterus in other species of rodents (Lv and Shi 2011). However, such 
changes to the uterus are not found in all rodents treated with these fertility compounds. Zhao et al. (2007) reported no significant differences in ovaries and uteri of Brandt's voles treated with quinestrol, levonorgestrel and their combination, whereas Liu et al. (2013) found reduced weight of ovaries but not uteri of Rattus nitidus treated with quinestrol. Lv and Shi (2011) also reported increased gonadosomatic indices of uteri and reduced gonadosomatic indices of ovaries after quinestrol treatment in Mongolian gerbils. Lv and Shi (2012) reported that quinestrol increases the weight of the uterus, while the ovary weight remained unchanged in young females borne from quinestrol-treated mothers, but not in levonorgestrel-treated mothers of Mongolian gerbils. These inconsistent findings in female reproductive organs might be caused by interspecies differences of oestrogen and progesterone sensitivity in the reproductive organs of different species of rodents as exemplified by Lv and Shi (2011). Furthermore, Huo et al. (2006) reported changes in uterine structure in more than $50 \%$ of female Mongolian gerbils treated with $1 \mathrm{mg} / \mathrm{kg}$ (1 ppm) body weight of quinestrol/levonorgestrel combination, and that the uteri were severely disrupted by higher dosages.

In M. natalensis the fertility compounds affected both pregnancy and litter size, with the most effective compound in reducing the number of pregnancies being quinestrol. Fertility control was effective when both sexes had been fed on treated bait although there were no significant differences between untreated females paired with treated males and treated females paired with treated males. If both sexes were treated with the different concentrations of each compound no pregnancies were observed, whereas when both sexes were left untreated the average litter size was four pups. In our pairing study, sample size was relatively low $(n=3)$ and further replication will help confirm these observed effects. Evidence from other studies on other rodent species does support our results. Field studies in China indicated that reduced pregnancy and litter size per pregnancy was reduced by $60 \%$ in the Campbell's dwarf hamster (Phodopus campbelli) treated with $0.01 \%$ quinestrol + levonorgestrel combination (Wan et al. 2006). Studies on other species of rodents have shown that there was reduced pregnancy rate and litter size in female Brandt's vole paired with treated males (Wang et al. 2011). Lv and Shi (2012) found reduced litter size of females from quinestrol-treated mothers in contrast to levonorgestrel-treated mothers. Liang et al. (2006) confirmed the effectiveness of quinestrol + levonorgestrel combination in reducing fertility in male and female Mongolian gerbils.

Oestrogenic compounds routinely used in agriculture, livestock production and for human contraception are leading to environmental contamination. Thus, registration authorities in some parts of the world are sceptical about using hormones and particularly oestrogenic compounds that may contribute to environmental contamination and adverse effects on non-target species including humans. However, not all endocrine disruptors are the same, and some research indicates that quinestrol and levonorgestrel decompose quickly under field conditions, with half-lives of 5-16 days in soil and less than 3 days in water (Tang et al. 2012a, b; Zhang et al. 2014). Considering the wellknown risks of many rodenticides in the environment and increased restrictions on their use, the registration of fertility control products for rodent pest management should be considered. Contraceptive baits would not be appropriate in all situations and unlikely to be acceptable in many household and urban situations; however, limiting rodent reproduction in areas where population irruptions occur could be highly sustainable with minimal non-target effects.

This study is the first to report on the effects of antifertility compounds on an outbreaking rodent pest species found throughout sub-Saharan Africa. The anti-fertility effects of synthetic steroid hormones (quinestrol and levonorgestrel) in rats have shown potential to control other species of rodents (Lv and Shi 2011; Liu et al. 2013). From our preliminary studies in the laboratory, the effects on fertility of $M$. natalensis seem very promising for field experimentation to reduce populations of this species which can have litter sizes of 3-17 pups and often reach $>200$ animals per hectare in crop fields (Coetzee 1965; Makundi and Massawe 2011). The next steps in this research would be to carry out bait feeding choice tests and field studies comparing the use of baits with rodenticide and anti-fertility compounds on the population dynamics of $M$. natalensis that also monitor agricultural crop damage levels.

\section{Author contributions}

SRB, RHM, ZZ, AWM, HJL and ML conceived and designed the experiments; GM, AW and RHM performed the experiments; GM, AWM and SRB analysed the data; and AWM, RHM, SRB, ZZ, GM, ML and HJL wrote the paper.

Acknowledgements The authors would like to thank those who helped carry out the experiments: Mrs. Mariam Kitojo, Resta Maganga, Mr. C. Pangapanga, Dr. Abdul Katakweba and Prof. L.S. Mulungu and two anonymous referees for helpful comments on the manuscript.

Funding This study was funded by a grant from the Agricultural Technology Transfer Programme from the United Kingdom's Department for International Development and partially supported by the External Cooperation Programme of BIC, Chinese Academy of 
Sciences, Grant No. 152111KYSB20150023. The funder had no role in study design, data collection and analysis, decision to publish, or in preparation of the manuscript.

\section{Compliance with ethical standards}

Conflict of interest The authors declare that they have no conflict of interest.

Ethical approval The study was conducted in laboratories of the Pest Management Centre, Sokoine University of Agriculture, Morogoro, Tanzania. All trials were approved by the university's ethics committee and followed the guidelines of the American Society of Mammalogists (Sikes and Gannon 2011).

Open Access This article is distributed under the terms of the Creative Commons Attribution 4.0 International License (http://crea tivecommons.org/licenses/by/4.0/), which permits unrestricted use, distribution, and reproduction in any medium, provided you give appropriate credit to the original author(s) and the source, provide a link to the Creative Commons license, and indicate if changes were made.

\section{References}

Asa CS, Porton IJ (eds) (2005) Wildlife contraception: issues methods and applications. John Hopkins University Press, Baltimore

Barlow ND (2000) The ecological challenge of immunocontraception. J Appl Ecol 37:897-902

Bomford M (1990) A role for fertility control in wildlife management. Australian Government Publishing Service, Canberra

Bordes F, Blasdell K, Morand S (2015) Transmission ecology of rodent-borne diseases: new frontiers. Integr Zool 10:424-435

Brown PR, Singleton GR, Kearns B, Griffiths J (1997) Evaluation and cost-effectiveness of strychnine for control of populations of wild house mice (Mus domesticus) in Victoria. Wildl Res 24:159-172

Brown PR, Chambers LK, Singleton GR (2002) Pre-sowing control of house mice (Mus domesticus) using zinc phosphide: efficacy and potential non-target effects. Wildl Res 29:27-37

Buckle AP, Smith RH (2015) Rodent pests and their control, 2nd edn. CABI, Wallingford

Coetzee CG (1965) The breeding season of the multimammate mouse Praomys (Mastomys) natalensis (A. Smith) in the transvaal highveld. Zool Afr 1:29-39

Fagerstone KA (2002) Wildlife fertility control. Wildl Soc Tech Rev 2:1-29

Fiedler LA (1988) Rodent pest problems and management in eastern Africa. FAO Plant Prot Bull 36:125-134

Fu H, Zhang J, Shi D, Wu X (2013) Effects of levonorgestrelquinestrol (EP-1) treatment on Mongolian gerbil wild populations: a case study. Integr Zool 8:277-284

Gemzell-Danielsson K, Marions L (2004) Mechanisms of action of mifepristone and levonorgestrel when used for emergency contraception. Hum Reprod Updat 10:341-348

Gonzales GF (2001) Function of seminal vesicles and their role on male fertility. Asian J Androl 3:251-258

Hone J (1992) Rate of increase and fertility control. J Appl Ecol 29:695-698

Humphrys S, Lapidge SJ (2008) Delivering and registering speciestailored oral antifertility products: a review. Wildl Res 35:578

Huo X, Wang D, Liang $\mathrm{H}$ et al (2006) A preliminary study on the anti-fertility effect of two sterilants to clawed jirds (Meriones unguiculatus). Acta Agrestia Sin 14:185-187
Jacob J, Herawati NA, Davis SA, Singleton GR (2004) The impact of sterilized females on enclosed populations of ricefield rats. J Wildl Manage 68:1130-1137

Jacob J, Singleton GR, Hinds LA (2008) Fertility control of rodent pests. Wildl Res 35:487

Katakweba AAS, Mulungu LS, Eiseb Seth J et al (2012) Prevalence of haemoparasites, leptospires and coccobacilli with potential for human infection in the blood of rodents and shrews from selected localities in Tanzania, Namibia and Swaziland. Afr Zool 47:119-127

Kirkpatrick JF, Turner JW (1985) Chemical fertility control and wildlife management. Bioscience 35:485-491

Leirs H (1992) Population ecology of mastomys natalensis (Smith, 1834) multimammate rats: possible implications for rodent control in Africa. Universitaire Instelling Antwerpen, Antwerp

Leirs H (1995) Population ecology of Mastomys natalensis (Smith, 1834). Implications for rodent control in Africa. A report from the Tanzania-Belgium joint rodent research project (1986-1989). Belgian Administration for Development Cooperation, Brussels

Leirs H, Verhagen R, Verheyen W (1994) The basis of reproductive seasonally in Mastomys rats (Rodentia: Muridae) in Tanzania. J Trop Ecol 10:55-66

Leirs H, Verhagen R, Sabuni CA et al (1997) Spatial dynamics of Mastoymys natalensis in a field-fallow mosaic in Tanzania. Belgian J Zool 127:29-38

Li J, Chen F, Li C, Chen Y (2014) Quinestrol induces spermatogenic apoptosis in vivo via increasing pro-apoptotic proteins in adult male mice. Tissue Cell 46:318-325

Liang H, Huo X, Wang D (2006) A preliminary study on the control effect of the sterilant on the population of clawed gerbil. Plant Prot 32(2):45-48

Liu M, Wan X, Yin Y et al (2012) Subfertile effects of quinestrol and levonorgestrel in male rats. Reprod Fertil Dev 24:297-308

Liu Q, Qin J, Chen Q et al (2013) Fertility control of Rattus nitidus using quinestrol: effects on reproductive organs and social behavior. Integr Zool 8:9-17

Liu M, Luo R, Wang H (2016) Recovery of fertility in quinestrol- or diethylstilbestrol (DES)-treated mice: implications for rodent management. Integr Zool 12(3):250-259

Lv X-H, Shi D-Z (2011) Effects of quinestrol as a contraceptive in mongolian gerbils (Meriones unguiculatus). Exp Anim 60:489-496

Lv X, Shi D (2012) Combined effects of levonorgestrel and quinestrol on reproductive hormone levels and receptor expression in females of the Mongolian gerbil (Meriones unguiculatus). Zool Sci 29:37-42

Lv X, Guo Y, Shi D (2012) Effects of quinestrol and levonorgestrel on prolactin serum concentration in lactating Mongolian gerbils (Meriones unguiculatus) and reproductive parameters of their offspring. Reprod Biol 12:285-292

Makundi RH, Massawe AW (2011) Ecologically based rodent management in Africa: potential and challenges. Wildl Res 38:588

Makundi RH, Bekele A, Leirs H et al (2005) Farmer's perceptions of rodents as crop pests: knowledge, attitudes and practices in rodent pest management in Tanzania and Ethiopia. Belgian $\mathbf{J}$ Zool 135:153-157

Massawe AW, Mulungu LS, Makundi RH et al (2011) Spatial and temporal population dynamics of rodents in three geographically different regions in Africa: implications for ecologically-based. Afr Zool 46:393-405

Massei G, Cowan D (2014) Fertility control to mitigate humanwildlife conflicts: a review. Wildl Res 41:1-21

Meerburg BG, Singleton GR, Kijlstra A (2009) Rodent-borne diseases and their risks for public health. Crit Rev Microbiol $35: 221-270$ 
Morand S, Bordes F, Chen H-W et al (2015) Global parasite and Rattus rodent invasions: the consequences for rodent-borne diseases. Integr Zool 10:409-423

Mulungu LS, Mahlaba TA, Masawe AW et al (2011) Dietary differences of the multimammate mouse, Mastomys natalensis (Smith, 1834), across different habitats and seasons in Tanzania and Swaziland. Wildl Res 38:640-646

Mwanjabe PS, Sirima FB, Lusingu J (2002) Crop losses due to outbreaks of Mastomys natalensis (Smith, 1834) Muridae, Rodentia, in the Lindi Region of Tanzania. Int Biodeterior Biodegrad 49:133-137

Nave CD, Coulson G, Short RV et al (2002) Long-term fertility control in the kangaroo and the wallaby using levonorgestrel implants. Reprod Suppl 60:71-80

Ngowo V, Mulungu LS, Lodal J et al (2005) Evaluation of thiram and cinnamamide for protection of maize seeds against multimammate mice, Mastomys natalensis, in Tanzania. Belgian J Zool 135:179-181

Novikova N, Weisberg E, Stanczyk FZ et al (2007) Effectiveness of levonorgestrel emergency contraception given before or after ovulation-a pilot study. Contraception 75:112-118

O'Donnell L, Robertson KM, Jones ME, Simpson ER (2001) Estrogen and spermatogenesis. Endocr Rev 22:289-318

Qu J, Liu M, Yang M et al (2015) Effects of fertility control in plateau pikas (Ochotona curzoniae) on diversity of native burds on Tibetan Plateau. Acta Theriol Sin 35:165-169

Singleton GR, Petch DA (1994) A review of the biology and management of rodent pests in southeast Asia. ACIAR Monogr Ser 30:1-65

Singleton GR, Brown PR, Jacob J, Aplin KP (2007) Unwanted and unintended effects of culling: a case for ecologically-based rodent management. Integr Zool 2:247-259

Singleton GR, Belmain SR, Brown PR, Hardy B (2010) Rodent outbreaks: ecology and impacts. Internatonal Rice Research Institute, Los Banos

Sridhara S, Dubey OP (2006) Rodent pest management in cultivated crops of India. Vertebr pests Agric Indian Scenar 131-192

Stenseth NC, Leirs H, Mercelis S, Mwanjabe P (2001) Comparing strategies for controlling an African pest rodent: an empirically based theoretical study. J Appl Ecol 38:1020-1031
Stenseth NC, Leirs H, Skonhoft A et al (2003) Mice, rats, and people: the bio-economics of agricultural rodent pests. Front Ecol Environ 1:367-375

Tang T, Qian K, Shi T et al (2012a) Photodegradation of quinestrol in waters and the transformation products by UV irradiation. Chemosphere 89:1419-1425

Tang T, Shi T, Li D et al (2012b) Adsorption properties and degradation dynamics of endocrine-disrupting chemical levonorgestrel in soils. J Agric Food Chem 60:3999-4004

Tuyttens FAM, Macdonald DW (1998) Fertility control: an option for non-lethal control of wild carnivores? Anim Welf 7:339-364

Wan X, Shi Y, Bao X et al (2006) Effect of the contraceptive compound (EP-1) on reproduction of the Djungarian hamster (Phodopus campbelli) in the typical steppe. Acta Theriol Sin 4:392-397

Wang D, Li N, Liu M et al (2011) Behavioral evaluation of quinestrol as a sterilant in male Brandt's voles. Physiol Behav 104:1024-1030

WHO (2010) WHO laboratory manual for the examination and processing of human semen. World Health Organization, Geneva

Williams CK, Davey CC, Moore RJ et al (2007) Population responses to sterility imposed on female European rabbits. J Appl Ecol 44:291-301

Zhang Z (2000) Mathematical models of wildlife management by contraception. Ecol Modell 1-2:105-113

Zhang Z (2015) A review on anti-fertility effects of levonorgestrel and quinestrol (EP -1) compounds and its components on small rodents. Acta Theriol Sin 35:203-210

Zhang Z, Wang Y, Wang S et al (2005) Effect of a contraceptive compound on reproduction of greater longtailed hamsters (Tscherskia triton) in experimental enclosures. Acta Theriol Sin 25:269-272

Zhang Q, Wang C, Liu W et al (2014) Degradation of the potential rodent contraceptive quinestrol and elimination of its estrogenic activity in soil and water. Environ Sci Pollut Res 21:652-659

Zhao M, Liu M, Li D et al (2007) Anti-fertility effect of levonorgestrel and quinestrol in Brandt's voles (Lasiopodomys brandtii). Integr Zool 2:260-268 